\title{
Hospital admission for acute stroke: who, for how long, and to what effect?
}

\author{
DERICK T WADE AND RICHARD LANGTON HEWER \\ From the Bristol Stroke Unit, Department of Neurology, Frenchay Hospital, Bristol BS16 $1 L E$
}

SUMMARY Data from 976 patients registered in a community survey as suffering an acute stroke have been analysed to discover what factors are associated with admission to hospital, how long patients spend in hospital, and whether there are any specific benefits attributable to hospital admission. Six hundred and twenty-five patients were admitted on account of their index stroke. These patients were compared with the 249 who remained at home throughout the first six months after the stroke-the remaining patients were admitted for other reasons or had their stroke in hospital. Two major factors related to an increased chance of admission: having a more severe disability and not having a carer. The same factors were associated with a longer length of stay for those who returned home within six months. Nevertheless $26 \%$ of patients managed at home were severely disabled when first seen within seven days. Patients admitted had a median stay of seven days if they died, 19 days if they returned home, and 149 days if they needed alternative long-term accommodation. Patients from the study population of 215000 people occupied an average of 11.4 beds/100 000 over the first six months after their stroke. After making allowance for the increased severity of disability seen in those admitted to hospital, no differences in functional, social or emotional outcome were found for either place of care.

Every day in 1982 our district general hospital spent $£ 1500$ caring for patients with acute stroke. ${ }^{1}$ This is comparable with the cost of stroke found in Scotland in $1974,{ }^{2}$ and it seems that between $4 \%$ and $5.6 \%$ of the total National Health Service (NHS) budget is devoted to caring for patients with acute stroke. A recent trial of an augmented home care service attempted to reduce the cost of stroke by encouraging general practitioners (GPs) to manage more patients at home, and by encouraging a more rapid discharge of patients from hospital. No effect was found in this matched controlled study of the patients under the care of two groups of GPs. ${ }^{3}$ Before trying other ways of reducing the cost of stroke to the NHS, it seems wise to study the use of hospitals in an attempt to identify potential ways of reducing their use.

In Manchester, ${ }^{4}$ three factors related to admission to hospital: the severity of the stroke, the degree of support available at home, and the apparent urgency engendered by the stroke. In a North London hospital, the length of stay was influenced by old age, loss of consciousness at onset, incontinence, and severity of stroke disability. ${ }^{5}$ Women may spend longer in hospital, ${ }^{16}$ possibly because fewer return home. Those who die occupy the least time whereas those needing long-term care occupy the most. ${ }^{17}$
The papers just reviewed give some information, but more research is needed because they concern selected patients: two-week survivors; 4 only those admitted to hospital $; 5$ or patients in other countries. ${ }^{67}$ To the best of our knowledge, no recent study has included all stroke patients from a defined population to investigate the comparative effectiveness of home and hospital care.

In this paper we wish to provide a foundation for developing more cost-effective ways of managing stroke by considering the following questions:

1 What factors relate to admission after an acute stroke?

2 How long do patients stay, and what influences this?

3 Are there any differences in outcome attributable to the place of care?

4 Do our findings suggest ways of improving the pattern of care offered stroke patients?

\section{Patients and methods}

This report is based on a series of 976 acute strokes registered in 215000 patients cared for by 96 GPs in the Frenchay Health District, Bristol. This district contains a mixture of urban and suburban patients, one town (Yate/Chipping Sodbury), and some 
isolated small villages and farms. Patients were notified by GPs, hospital staff, and anyone else involved in stroke care (eg, district nurses). Hospital patients were checked in several ways: wards were visited regularly to seek out patients, therapists and others were constantly reminded to notify patients, and the Regional Health Authority gave us the record numbers of all patients discharged from hospitals in Avon county with a primary or secondary diagnosis of stroke. The notes of these patients were obtained, and patients were registered if they had had a stroke and were from our study population. Every patient suffering an acute stroke between 1 March 1981 and 30 June 1983 has been included. Further strokes were re-registered only if over six months had elapsed from the index stroke $(n=17)$.

Stroke was a clinical diagnosis based on the WHO definition. ${ }^{8}$ It was confirmed by a neurologist's examination (DW) in 713 (73\%) cases, the remaining diagnoses being based on information gained from hospital notes. For patients admitted to hospital during the first six months after a stroke, a judgement was made as to the reason for their admission. In cases of doubt it was assumed that the stroke caused admission. Analysis will compare patients admitted for their index stroke with those who were never admitted, leaving out those who were already in hospital and those admitted for a second stroke or for other reasons.

Patients were assessed as soon as possible, at three weeks and at six months post-stroke. Physical function was assessed using the Barthel Activity of Daily Living (ADL) scale, ${ }^{9}$ which is probably equivalent to most other ADL scales, ${ }^{10}$ measuring independence on 10 activities (eg, dressing, walking) and giving a score between 0 and 20 in 1 point increments - a score of 20 implies independence but not necessarily normality. Depression was measured using the Wakefield self-assessment depression

Table 1 Characteristics of patients

\begin{tabular}{lrr}
\hline Group & $\begin{array}{l}\text { Admitted } \\
n=625\end{array}$ & $\begin{array}{l}\text { Not admitted } \\
n=249\end{array}$ \\
\hline Male & $286(46 \%)$ & $124(50 \%)$ \\
Average age (SD) & $71 \cdot 7(12 \cdot 4)$ & $73 \cdot 3(9 \cdot 0)$ \\
Side of weakness: & $296(47 \%)$ & $103(41 \%)$ \\
$\quad$ Right & $255(41 \%)$ & $101(41 \%)$ \\
$\quad$ Left & $72(12 \%)$ & $32(13 \%)$ \\
'Both/neither' & $2(<1 \%)$ & $13(5 \%)$ \\
Unknown & & \\
$\begin{array}{l}\text { X } 1 \cdot 26 ; \text { NS } \\
\text { Previous disability (Barthel ADL score) }\end{array}$ & $1(<1 \%)$ & $0(0 \%)$ \\
$0-4$ (very severe) & $3(1 \%)$ & $2(1 \%)$ \\
$5-9$ (severe) & $25(4 \%)$ & $5(2 \%)$ \\
$10-14$ (moderate) & $112(18 \%)$ & $33(13 \%)$ \\
$15-19$ (mild) $\quad$ (normal) & $420(67 \%)$ & $189(76 \%)$ \\
$20 \quad 64(10 \%)$ & $20(8 \%)$ \\
Not known & & \\
$X=6 \cdot 2 ;$ NS & & \\
\hline
\end{tabular}

inventory, ${ }^{11}$ a scale with 12 statements giving a score between 0 and 36; any score of 15 or more signifies "depression". Social function was measured using the Frenchay Activities Index (FAI); ${ }^{12}$ a patient scores between 0 and 45, depending upon the frequency with which he performs each of 15 separate activities (eg, washing up, going for walks). Language function (aphasia) was assessed in four parts-comprehension, expression, reading, writing-using three tests from the "Schuell"13 and a reading test from the "Boston". ${ }^{14}$ Each test was scored from 0-5, giving a total score range of 0-20. Carers (ie, the person, usually a relative, who lived with and gave support to the patient) were assessed using the General Health Questionnaire (GHQ). ${ }^{15}$

\section{Results}

Over the first six months post-stroke, $249(26 \%)$ of the 976 patients remained at home, $625(64 \%)$ were admitted directly on account of their stroke, $67(7 \%)$ were already in hospital, $26(3 \%)$ were admitted for reasons unrelated to their stroke, and 9 were admitted with a further stroke. Those admitted were slightly younger $(t=2.09 ; \mathrm{p}<0.02)$ : this and some other characteristics of the two groups are shown in table 1. Factors that were NOT associated with admission to hospital included: previous stroke, presence of telephone in the house, the type of housing (ownership or structure), location of patient when stroke occurred (eg, at home, in a public place), and previous social activities (FAI scores).

The influence of the mode of onset, social support, and the initial severity of stroke is shown in table 2 .

Table 2 Factors associated with admission to hospital

\begin{tabular}{|c|c|c|}
\hline Group & $\begin{array}{l}\text { Admitted } \\
n=625\end{array}$ & $\begin{array}{l}\text { Not admitted } \\
n=249\end{array}$ \\
\hline \multicolumn{3}{|c|}{ Loss of consciousness at onset: } \\
\hline None & $266(43 \%)$ & $163(66 \%)$ \\
\hline Asleep & $68(11 \%)$ & $49(20 \%)$ \\
\hline Slight/delayed LoC & $141(23 \%)$ & $11(4 \%)$ \\
\hline Immediate LoC & $134(21 \%)$ & $10(5 \%)$ \\
\hline Not known & $16(3 \%)$ & $12(5 \%)$ \\
\hline \multicolumn{3}{|l|}{$\chi=97.3 ; p<0.001$} \\
\hline \multicolumn{3}{|l|}{ Home support: } \\
\hline Lives alone & $170(27 \%)$ & $33(13 \%)$ \\
\hline Carer not fit & $53(9 \%)$ & $10(4 \%)$ \\
\hline Carer works & $82(13 \%)$ & $30(12 \%)$ \\
\hline Carer able & $275(44 \%)$ & $153(61 \%)$ \\
\hline Institutional carers & $40(6 \%)$ & $19(8 \%)$ \\
\hline Unknown & $5(1 \%)$ & $4(2 \%)$ \\
\hline \multicolumn{3}{|c|}{$\begin{array}{l}\chi=31 \cdot 2 ; p<0 \cdot 001 \\
\text { Barthel ADL score (first week): }\end{array}$} \\
\hline Number & $n=337$ & $n=159$ \\
\hline $0-4$ (very severe) & $170(50 \%)$ & $22(14 \%)$ \\
\hline $5-9$ (severe) & $79(23 \%)$ & $19(12 \%)$ \\
\hline 10-14 (moderate) & $43(13 \%)$ & $33(21 \%)$ \\
\hline $15-19$ (mild) & $30(10 \%)$ & $41(26 \%)$ \\
\hline 20 (normal) & $15(4 \%)$ & $44(27 \%)$ \\
\hline \multicolumn{3}{|l|}{$x=119.6 ; p<0.001$} \\
\hline
\end{tabular}


The ADL results relate only to those patients assessed within the first seven days of stroke. The close association between disability and admission is obvious, and this association was apparent, however that severity was measured: for example, those admitted not only had more severe paralysis but also were more likely to be in coma, have a hemianopia, be incontinent, and to have lost sitting balance. Nevertheless $26 \%$ of those managed at home were severely disabled (Barthel score 9 or less) in the first few days. Although loss of consciousness was associated with admission, the speed of stroke onset was NOT associated with admission.

Aphasia was associated with hospital admission. Considering only those who were conscious and who were seen within seven days, 60 (30\%) of 200 patients admitted had no comprehension (ie, did not point to two objects in a picture when asked to) but only $13(9 \%)$ of those at home were so severely affected. This difference was also found for expression. Further analysis showed that those with aphasia were more disabled: considering only patients seen within seven days, those with an 'aphasia score' of 14 or less had an average (SD) Barthel score of $8.4(6.9)$ as compared with 12.8 $(5 \cdot 8)$ for those with intact language $(t=6 \cdot 16$; $\mathrm{p}<0.001)$.

The majority $(385 ; 62 \%)$ of admissions were instigated by the GP, a further $66(11 \%)$ being arranged by the Doctor's Deputising Service and 37 $(6 \%)$ after consultant domiciliary visits or outpatient appointments. One hundred and seven $(17 \%)$ of admissions were directly from home through casualty, and the remaining 30 were through casualty from elsewhere. Most $(488 ; 79 \%)$ admissions were within one day of the stroke, with a further $51(8 \%)$ on the second and third days. Only $50(8 \%)$ of admissions occurred after the first week.

A majority $(347 ; 55 \%)$ of patients were admitted to the District General Hospital (Frenchay); a further $90(14 \%)$ went to a local small hospital (Cossham) which accepts medical emergencies, and $102(16 \%)$ were admitted to the local geriatric hospital (Manor Park). Eighty-six (13\%) patients were admitted to hospitals outside the district. By six months post-stroke, most patients had either died in the hospital to which they were first admitted (282; $45 \%)$ or had returned home $(259 ; 41 \%), 38(6 \%)$ being transferred to another hospital, $30(5 \%)$ leaving for a new home, and $16(3 \%)$ being still in their first hospital.

The length of stay over the first six months (183 days) post-stroke was calculated for each patient's first hospital stay: if a patient moved directly to another hospital, that time was included but readmissions were excluded -44 were readmitted at
Table 3 Length of first stay in days according to outcome

\begin{tabular}{llll}
\hline First discharge & Died & Returned home & Other \\
\hline Number & 293 & 266 & 66 \\
Average (SD) & $17 \cdot 2(25 \cdot 0)$ & $29 \cdot 6(30 \cdot 1)$ & $127(63 \cdot 7)$ \\
Range & $1-160$ & $2-182$ & $4-183$ \\
Median & 7 & 19 & 149 \\
Categories: & & & \\
$\quad$ 0-7 days & $148(50 \%)$ & $45(17 \%)$ & $2(3 \%)$ \\
$8-14$ days & $55(19 \%)$ & $59(22 \%)$ & $1(2 \%)$ \\
15-28 days & $38(13 \%)$ & $73(28 \%)$ & $5(7 \%)$ \\
29-56 days & $32(11 \%)$ & $49(18 \%)$ & $7(11 \%)$ \\
Longer & $20(7 \%)$ & $40(15 \%)$ & $51(77 \%)$ \\
\hline
\end{tabular}

least once within six months of their stroke. Table 3 shows the length of stay for three groups of patients: those returning home, those dying, and those going elsewhere. Over the first six months, 20915 bed-days were used, of which $24 \%$ were devoted to those dying in their first hospital stay, $38 \%$ to those going home, and $38 \%$ to the remaining patients. Considered in relation to the study population of 215000 , these figures represent an average need for 11.4 beds $/ 100000$ devoted to the first six months' care of patients admitted with acute stroke. No account has been taken of the need for long-term (over six months) care or for holiday relief admissions. These needs will be met in different ways in different communities.

The most obvious factor related to the length of stay was the severity of stroke induced disability. Considering only those patients first seen within a week of their stroke and who finally returned home $(n=158)$, the correlation coefficient between their Barthel score and length of stay was -0.519 $(p<0 \cdot 01)$. Most other factors indicative of stroke severity were correlated with length of stay; for example, those found to be incontinent of urine when first seen within seven days of their stroke $(n=54)$ had an average stay of $40 \cdot 2$ days (SD 26.7) but the 104 continent patients had a stay of $26 \cdot 8$ days (SD $29.5 ; t=2.88 ; \mathrm{p}<0.01)$. Support at home also influenced the length of stay: 57 patients with no carer at home had an average (SD) stay of 39.1 (39.9) days as compared with $26 \cdot 8(25 \cdot 3)$ days for the 182 patients with some form of support $(t=2 \cdot 21$; $\mathrm{p}<0.01)$. Neither the sex of the patient nor the side of stroke induced weakness influenced the length of stay of those going home, but (old) age was correlated with length of stay of those going home $(\mathrm{r}=+0.205 ; \mathrm{p}<0.01)$.

Most patients had some investigations, although a few died so rapidly that none was performed. Information was available on 565 of the 625 patients admitted: $527(93 \%)$ had a full blood count, 421 $(75 \%)$ a chest $x$ ray, and $392(69 \%)$ a blood glucose estimation. Neurological investigations were less frequent: $190(34 \%)$ had a CT scan, $101(18 \%)$ a 
skull $x$ ray, $33(6 \%)$ a lumbar puncture, $19(3 \%)$ an angiogram, $7(1 \%)$ an electroencephalogram, and 3 (1\%) an isotope brain scan. Apart from the 402 (71\%) who had an electrocardiogram, few had specific cardiac investigations: $78(14 \%)$ had their cardiac enzymes measured, and $16(3 \%)$ an echocardiogram. All of these investigations are easily available locally, particularly for the 347 admitted to Frenchay Hospital.

By six months post-stroke, $47(19 \%)$ of those managed at home and $332(54 \%)$ of those admitted had died (some at home). Considering survivors, 224 $(76 \%)$ of those who had been admitted and 195 (97\%) of those not admitted were living in their original homes. Forty-eight (16\%) of those admitted were still in hospital or in a nursing home, $11(4 \%)$ were in sheltered accommodation, 8 had moved in with relatives, and 2 had moved to a new house. Of those managed at home, 4 had moved house and 2 had entered sheltered accommodation.

Analysis of the outcome at six months is complicated by the fact that those admitted had a more severe initial disability. In order to overcome this, two-way analysis of variance has been used, dividing patients first by disability and second by place of care. Considering survival and ADL outcome (table 4), patients were divided by their Barthel score when first seen within seven days: patients seen later or not seen have been excluded from this analysis. Allowing for the severity of stroke, there was no difference in the mortality rates $(\chi=0.57 ; \mathrm{NS})$. Two-way analysis of variance confirms that initial severity does influence final ADL ability $(F=36.17 ; p<0.01)$ but showed that place of care did not $(\mathrm{F}=0 \cdot 29$; NS).

Social activities and depression may also be influenced by disability, and so two-way analysis of variance was used in analysing these outcomes. For social function, as measured by FAI scores, patients who were living at home were divided first by place of care (home/hospital) and then into three ADL

Table 4 Outcome according to initial severity and place of care

\begin{tabular}{llll}
\hline & \multicolumn{4}{l}{ Initial Barthel score } \\
\cline { 2 - 4 } Outcome at 6 months & $0-9$ & $10-18$ & $19-20$ \\
\hline Admitted & 249 & 66 & 22 \\
Died & $130(52 \%)$ & $15(23 \%)$ & $2(9 \%)$ \\
Lost to F-U & $2(1 \%)$ & $3(4 \%)$ & 0 \\
Alive & $117(47 \%)$ & $48(73 \%)$ & $20(91 \%)$ \\
Av (SD) Barthel score & $14 \cdot 0(5 \cdot 5)$ & $18 \cdot 4(3 \cdot 8)$ & $19 \cdot 7(0 \cdot 6)$ \\
At home & $41(59)$ & $59(7 \%)$ & $1(2 \%)$ \\
Died & $18(44 \%)$ & $2(3 \%)$ & $1(2 \%)$ \\
Lost to F-U & 0 & $57(96 \%)$ \\
Alive & $23(56 \%)$ & $53(90 \%)$ & $57(96)$ \\
Av (SD) Barthel score & $14 \cdot 7(4 \cdot 4)$ & $18 \cdot 4(2 \cdot 8)$ & $19 \cdot 8(0 \cdot 5)$ \\
\hline
\end{tabular}

groups based on their Barthel score at six months: poor function (0-14), some disability (15-19), and no disability (20). This showed no influence of admission ( $\mathrm{F}=0 \cdot 128$; NS) but there was an obvious influence of disability $(F=116.46 ; p<0.01)$. Some patients could not complete the "Wakefield" depression scale because of aphasia or confusion, but in those who could, depression (score over 14) was present in $27 \%$ (54 of 165) of those managed at home and in $25 \%$ (37 of 146 ) of those originally managed in hospital but at home by six months; it was more common in those who had not returned home from hospital (16 of $33 ; 48 \%)$. Two-way analysis of variance confirmed that the place of care did not influence depression $(\mathrm{F}=0 \cdot 128 ; \mathrm{NS})$ but that disability did $(\mathrm{F}=7 \cdot 65 ; \mathrm{p}<0 \cdot 01)$.

Carers of all patients at home were assessed at six months. For those who had been in hospital, 23 $(24 \%)$ of 94 carers were depressed as compared with $21(18 \%)$ of 119 carers of those not admitted $(\chi=1 \cdot 1$; NS). There was no difference in the average Wakefield scores $(t=1 \cdot 17$; NS) or the average GHQ scores $(t=1.04$; NS). Finally, only nine carers of those admitted and six carers of those kept at home had to stop work: most either never had worked $(75 \%)$ or continued as before $(20 \%)$.

\section{Discussion}

This is the first British-based community survey of stroke to report in detail about the use of hospitals by patients with acute stroke. It confirms that admission is influenced by the severity of stroke and by social support at home, but it highlights the fact that not every severely disabled stroke patient is admitted to hospital in this country. Consequently hospital-based studies are likely to be incomplete and biased as they may neglect many less severe strokes and some severe strokes. This study confirms that stroke severity has an important influence on the length of stay of a patient in hospital and shows that the lack of a carer at home also prolongs hospital stay.

This study is the first to compare (statistically) the outcomes in those managed at home with the outcomes of those admitted to hospital. It should be stressed at this point that the results are observational. The data, however, were collected as part of a trial of a home care service for patients with acute stroke. ${ }^{3}$ As reference will be made to the results of the trial, a few points about that trial will be emphasised. It was not a classical randomised controlled trial, rather the patients registered with two groups of GPs were compared. Decisions concerning patient management were made independently by the doctor concerned, but one group of patients had access to additional home care. 
Thus the trial investigated the effect that the ready availability of additional home care had on management decisions of both GPs and hospital doctors (regarding discharge). The advantages and disadvantages of this study design have been discussed. ${ }^{3}$

The patients included in this study came from a defined population and should include almost all those admitted to hospital. It is obviously impossible to check on those not admitted but not notified, and so we may have missed some patients at home-indeed, this is quite likely as we had a higher notification from the trial group of GPs. ${ }^{3}$ Nevertheless we have probably included at least $80 \%$ of strokes as our incidence rate of $1.90 / 1000 /$ year is comparable with that in other published studies. ${ }^{8}$ The Health District is probably representative of many in Britain with a mixture of urban, suburban, and rural areas.

This study confirms earlier reports ${ }^{4}$ that those admitted are more likely to live alone and are more likely to have a more severe stroke. Aphasia was associated with a more severe functional (ADL) loss - this association has been noted before ${ }^{4}$ but our results suggest that it is probably secondary to the increased disability. Two other observations relating to admission are of interest. Firstly, about $10 \%$ of admissions occurred after the acute illness had resolved (ie, after four days). This implies that home management had been tried and failed, but the provision of (limited) extra support did not seem to reduce late admission. ${ }^{3}$ Secondly, $25 \%$ of admissions were "inevitable" in that patients arrived without first seeing a doctor, and a further $10 \%$ were seen only by a deputising doctor.

Based on the observations made in this study, it is difficult to propose any rational way of reducing the admission rate because providing more home support does not seem to be effective. ${ }^{3}$ Only a minority of patients had specialised investigations, suggesting that few were admitted for diagnostic reasons. There are parts of Britain with markedly lower admission rates. In the Oxfordshire Community Stroke Project ${ }^{16}$ only $40 \%$ of patients suffering their first stroke are admitted to hospital ${ }^{17}$ and this despite an almost complete absence of a local domiciliary physiotherapy service. The only other community based report to include an estimate of the admission rate ${ }^{18}$ suggested that $25 \%$ were at home at three weeks post-stroke. One doctor has suggested that as many as $60 \%$ of patients with acute stroke are managed at home. ${ }^{19}$ Thus it seems possible that there are variations in local admission policies of such a scale as to dwarf any minor changes which might be achieved by increasing home support or by other means. Further investigation of these differences is needed to establish whether they simply reflect differences in stroke severity and social support, and whether the outcome is different.

The length of stay of patients from our Health District would seem to be comparable with others, ${ }^{520}$ particularly when one considers the large variance seen in the average figures. About $25 \%$ of this variance is accounted for by the severity of the stroke, and the absence of a carer at home prolongs hospital stay, but there does not seem to be any other major influence on the length of stay. The lengths of stay in various hospitals in our District have not been compared because there are so many (unknown) selection factors influencing which hospital patients are admitted to (eg, the elderly socially isolated patient was more likely to be admitted to the hospital run by the geriatricians). The figures found in this study suggest that the average Health District of 250000 people will devote nearly 30 beds to the acute (first six months) care of patients admitted with stroke. This figure will not be influenced much by the local provision of nursing homes, respite admissions, etc, as these concern only patients with long term disabilities. These latter patients probably need about 20 beds each year. There are no British population based studies to compare this figure with, but an estimate from the USA found a need for 24 beds for a similar population.?

Again the observations made in this study do not suggest any way to reduce the use of beds. It is worth noting, however, that stroke severity and the absence of a carer do not account for all the variance seen in the lengths of stay, suggesting that other (possibly alterable) factors do influence the length of stay. We have previously shown ${ }^{21}$ that discharge after stroke may be unnecessarily delayed in some patients. Thus, although one American study ${ }^{22}$ has found that discharge planning does not consistently reduce the length of stay, a similar study in this country might show some benefit from more concentration on the process of hospital discharge.

Any analysis attempting to identify the effect of admission on outcome needs to take into account the fact that those admitted are more severely disabled and more likely to live alone, both factors which might influence outcome. We have used two-way analysis of variance to balance for the effect of stroke severity and found that there were no differences between the two groups in terms of functional or social recovery and adaptation. Equally, there does not appear to be any increased prevalence of depression among the carers of those kept at home. Thus, although those admitted were a selected group, the use of statistical techniques to control for some of the selection factors revealed that neither place of 
care (home or hospital) offered any great advantages either to the patient or to the family.

To conclude, this study shows that stroke patients who were more severely disabled by their stroke and those who lived alone were more likely both to be admitted to hospital and to spend longer in hospital before returning home. Patients from our Health District occupied an average of 25 beds at any one time purely in relation to their acute care over the first six months. Relatively few patients underwent specialised investigation in hospital, and no specific benefit has been shown for hospital (or home) care in terms of functional, social, or emotional adaptation by the patient or in terms of stress upon the carer. In order to reduce the cost of caring for stroke patients, it might be best to investigate the differences already existing between different parts of the country, and also the use of a more planned approach to hospital discharge.

We gratefully acknowledge the cooperation of all those involved in helping with the main project, including all the GPs, medical records departments, and all hospital staff. Financial support was given by the Department of Health and Social Security and Avon Joint Funding Committee.

\section{References}

${ }^{1}$ Wade DT, Wood VA, Langton Hewer R. Use of hospital resources by acute stroke patients. J Roy Coll Phys (Lond) 1985; 19: 48-52.

${ }^{2}$ Carstairs V. Stroke: resource consumption and the cost to the community. In: Gillingham FJ, Mawdsley C, Williams AE, eds. Stroke. Edinburgh: Churchill Livingstone, 1976; 516-28.

${ }^{3}$ Wade DT, Langton Hewer R, Skilbeck CE, Bainton D, Burns-Cox C. Controlled trial of a home-care service for acute stroke patients. Lancet 1985; i: 323-6.

${ }^{4}$ Brocklehurst JC, Andrews K, Morris P, Richards BR, Laycock PL. Why admit stroke patients to hospital? Age Ageing 1978; 7: 100-8.
${ }^{5}$ Sheikh K, Meade TW, Brennan PJ, Goldenberg E, Smith DS. Intensive rehabilitation after stroke: service implications. Community Medicine 1981; 3: 210-6.

${ }^{6}$ Terent A. Medico-social consequences and direct costs of stroke in a Swedish community. Scand J Rehab Med 1983; 15: 165-71.

${ }^{7}$ Gibson CJ. Epidemiology and patterns of care of stroke patients. Arch Phys Med Rehabil 1974; 55: 898-903.

${ }^{8}$ Aho K, Harmsen P, Hatano S, Marquardsen J, Smirnov V, Strasser T. Cerebrovascular disease in the community: results of a WHO collaborative study. Bull WHO 1980; 58: 113-30.

${ }^{9}$ Granger CV, Dewis LS, Peters NC, Sherwood CC, Barrett JE. Stroke rehabilitation: analysis of repeated Barthel index measures. Arch Phys Med Rehabil 1979; 60: 14-7.

${ }^{10}$ Gresham GE, Phillips TF, Labi MLC. ADL status in stroke: relative merits of three standard indexes. Arch Phys Med Rehabil 1980; 61: 355-8.

${ }^{11}$ Snaith RP, Ahmed SN, Mehta S, Hamilton M. Assessment of the severity of primary depressive illness: Wakefield self-assessment depression inventory. Psychol Med 1971; 1: 143-9.

${ }^{12}$ Holbrook M, Skilbeck CE. An activities index for use with stroke patients. Age Ageing 1983; 12: 166-70.

${ }^{13}$ Schuell H. The Minnesota test for the differential diagnosis of aphasia. Minneapolis: University of Minnesota Press, 1965.

${ }^{14}$ Goodglass $\mathrm{H}$, Kaplan E. The assessment of aphasia and related disorders. Philadelphia: Lea and Febiger, 1972.

${ }^{15}$ Goldberg DP, Hillier VF. A scaled version of the General Health Questionnaire. Psychol Med 1979; 9: 139-45.

${ }^{16}$ Oxfordshire Community Stroke Project. Incidence of stroke in Oxfordshire: first year's experience of a community stroke register. $\mathrm{Br}$ Med J 1983; 287: 713-6.

${ }^{17}$ Sandercock PAG. The Oxford Community Stroke Project and its application to stroke prevention. DM Thesis, University of Oxford, 1984.

${ }^{18}$ Weddell JM, Beresford SAA. Planning for stroke patients. A four year descriptive study of home and hospital care. Department of Health and Social Security. London: HMSO, 1979.

${ }^{19}$ Cochrane AL. Burden of cerebrovascular disease. Br Med $J$ 1970; 287: 165.

${ }^{20}$ Garraway WM, Akhtar AJ, Smith DL, Smith DE. The triage of stroke rehabilitation. J Epidemiol Community Health 1981; 35: 39-44.

${ }^{21}$ Wade DT, Wood VA, Langton Hewer R. Recovery after stroke: the first three months. J Neurol Neurosurg Psychiatry 1985; 48: 7-13.

${ }^{22}$ Cable EP, Mayers SP. Discharge planning effect on length of hospital stay. Arch Phys Med Rehabil 1983; 64: $57-60$. 